\title{
Nonlocal Wavefunction Collapse for a Single Particle Using Homodyne Measurement without Post-selection
}

\author{
Maria Fuwa, ${ }^{1}$ Shuntaro Takeda, ${ }^{1}$ Marcin Zwierz, ${ }^{2}$ \\ Howard Wiseman, ${ }^{3}$ and Akira Furusawa ${ }^{1}$ \\ ${ }^{1}$ Department of Applied Physics, School of Engineering, The University of Tokyo, 7-3-1 Hongo, Bunkyo-ku, Tokyo 113-8656, Japan \\ ${ }^{2}$ Faculty of Physics, University of Warsaw, 00-681 Warsaw, Poland \\ ${ }^{3}$ Centre for Quantum Dynamics and Centre for Quantum Computation and Communication Technology, Griffith University, \\ Brisbane, QLD 4111, Australia
}

\begin{abstract}
We demonstrate the nonlocal wavefunction collapse for a single particle, the idea of which dates back to Einstein's first argument on his concerns about quantum theory, by performing EPR-steering using heralded single photons.
\end{abstract}

(C) 2014 Optical Society of America

OCIS codes: (270.5585) Quantum information processing; (120.2920) Homodyning

A single quantum particle can have a wavefunction that spreads over arbitrarily large distances, but it is never detected at two places. This is explained in quantum theory by what Einstein called "spooky action at a distance": the instantaneous nonlocal collapse of its wavefunction to the place of detection. To test experimentally that a measurement in one lab (Alice's) really causes a change in the quantum state in a distant lab (Bob's) requires Alice to choose between different measurements, and observing the effect of this choice on Bob's state. Here we perform such a test for the first time with a single particle split between two parties. We verify Einstein's action at a distance for a single photon by employing homodyne measurement with six different phases to violate an Einstein-Podolsky-Rosen-steering (EPR-steering) inequality by $0.030 \pm 0.005$. This witnesses the entanglement of the split photon even when Alice is untrusted.

When Einstein first argued against the nonlocal collapse of the wavefunction [1], he considered just a single particle. The particle's wavefunction was diffracted through a tiny hole so that it "dispersed" over a large hemispherical area prior to encountering a screen of that shape covered in photographic film [Fig. 1 (a)]. Since the film only ever registers the particle at one point on the screen, orthodox quantum mechanics must postulate a "peculiar mechanism of action at a distance, which prevents the wave ... from producing an action in two places on the screen". That is, according to the theory, the detection at one point must instantaneously collapse the wavefunction to nothing at all other points.

As was proposed in [2], we put to test a simplified version of this Einstein's gedankenexperiment so that the heralded single photon is split into just two wavepackets, by impinging it on a beam splitter of reflectivity $R=0.50$ [Fig. 1 (b)]

$$
|\psi\rangle=\frac{1}{\sqrt{2}}\left(|0\rangle_{\mathrm{A}}|1\rangle_{\mathrm{B}}-|1\rangle_{\mathrm{A}}|0\rangle_{\mathrm{B}}\right) \text {. }
$$

One is sent to Alice and the other to Bob. But there is a key difference from Einstein's original conception, which enables us to demonstrate the nonlocal collapse experimentally, with minimal theoretical assumptions. That is to use
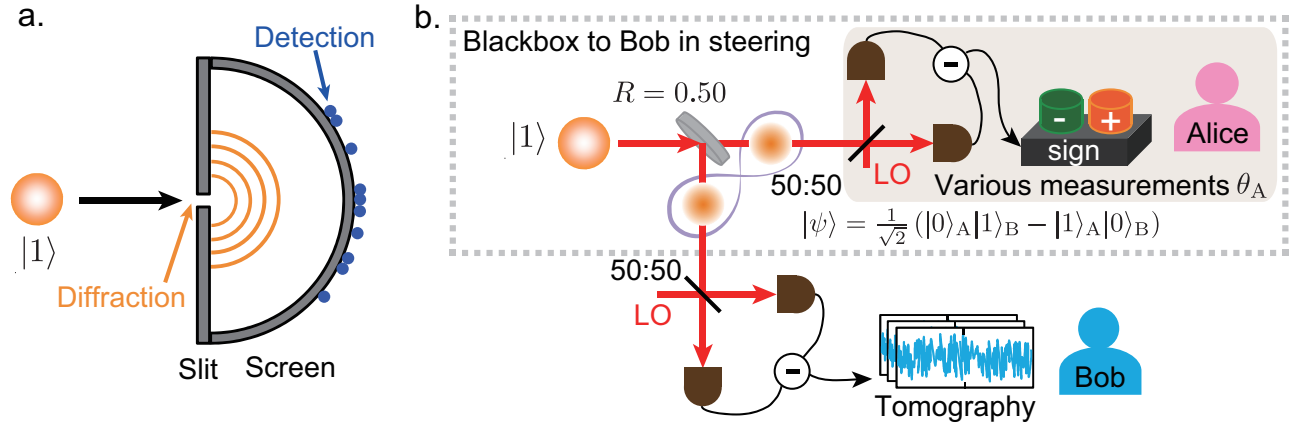

Fig. 1. (a) Einstein's proposal. (b) Overall schematic. LO; local oscillator. 
homodyne measurements to obtain complete information of the change in the state (i.e. tomography) at one location (Bob), conditioned on the measured values at the other (Alice), and also to give Alice the power to make different homodyne measurements. She does this by choosing the phase of her LO $\left(\theta_{\mathrm{A}}\right)$, which in our experiment is chosen at random from six possible values, $\theta_{\mathrm{A}}=0, \pm \pi / 6, \pm \pi / 3, \pi / 2$. When Alice gets result $x_{\theta_{\mathrm{A}}} \in \mathbb{R}$, she collapses Bob's local wavefunction to $|0\rangle-e^{-i \theta_{\mathrm{A}}} \sqrt{2} x_{\theta_{\mathrm{A}}}|1\rangle$. Thus by changing her LO phase $\theta_{\mathrm{A}}$, Alice controls the relative phase of the vacuum and one photon component of Bob's conditioned state, modulo $\pi$, depending on the sign of the $x$ she obtains. Because of this, it is convenient for Alice to coarse-grain her result to $s=\operatorname{sign}\left(x_{\theta_{\mathrm{A}}}\right) \in\{+,-\}$.

To reconstruct his unconditional and conditional local states, Bob performs homodyne measurements using a randomly chosen LO phase $\theta_{\mathrm{B}} \in[0,2 \pi]$ for each partial photon. The unconditional local states, reconstructed using Bob's total data, is a phase independent statistical mixture of vacuum and single photons [Fig. 2 (a)]. This is because when the detection is performed in only one location (Bob), the relative phase information between two spatially separated partial photon's (Alice and Bob) are lost. Now Bob divides his quadrature datum according to Alice's measurement bases $\theta_{\mathrm{A}}$ and results $s$, and uses the datum for each condition to reconstruct the corresponding conditional local states [Fig. 2 (b)-(d)]. Their clear phase dependence reveals that Bob's local wavefunction has collapsed to a superposition of vacuum and single photon. Since Bob's wavefunction collapses to a different superposition in consequence of Alice's projection choice of local oscillator, this suggests that it is impossible to assign a quantum state to Bob's system (part of a single photon) independent of Alice's measurement, and thus enables Bob alone to verify the nonlocality of the single photon.
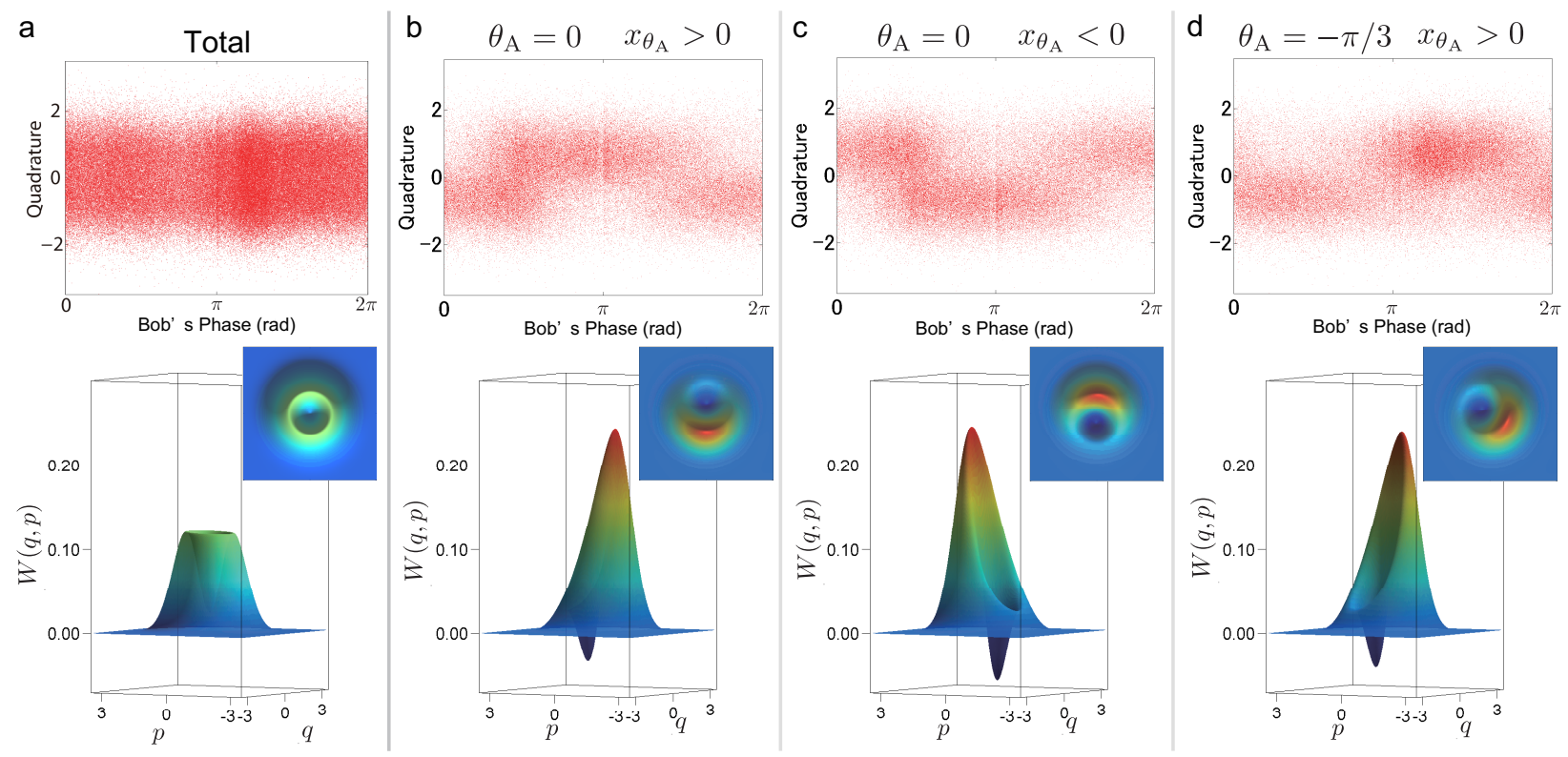

Fig. 2. Bob's local states. (a) Bob's unconditional states. (b)-(d) Bob's conditional states. (Top) Bob's marginal distributions. (Bottom) Wigner functions; top view in inset; $\hbar=1$.

Finally, we quantitatively confirm this form of quantum non-locality by violation of the EPR-steering inequality [2]. This is when the measured correlation function exceeds the limit allowed by a local quantum state model for Bob's system. The EPR-steering inequality calculated from Bob's unconditional and conditional local states was violated by $0.030 \pm 0.005$ (measured correlations: $0.672 \pm 0.002$, local limit: $0.6422 \pm 0.0001$ ).

In conclusion, we experimentally demonstrate the nonlocal wavefunction collapse for a single particle, the idea of which dates back to Einstein's first argument on his concerns about quantum theory, by performing EPR-steering using heralded single photons.

\section{References}

1. G. Bacciagaluppi and A. Valentini. Quantum Theory at the Crossroads: Reconsidering the 1927 Solvay Conference. Cambridge University Press (2009).

2. S. J. Jones, and H. M. Wiseman, Phys. Rev. A 84, 012110 (2011). 